Discussion paper

\title{
Implementing Care Groups for improved maternal and child nutrition: Critical factors for success from the Amalima program in rural Zimbabwe
}

\section{Authors:}

Pamela Ncube - Murakwani

Lead Nutrition Specialist, International Medical Corps

pmurakwani@yahoo.co.uk

Shamiso Alice Moyo

Public Health and Nutrition Specialist

shamyalice@yahoo.com

Craig Nyathi

Nutrition Coordinator, International Medical Corps

Mutsa Dzimba

Social Behaviour Change Nutritionist, The Manoff Group

Mackson Maphosa

Behaviour Change Specialist, The Manoff Group

Sijabulisiwe Beatrice Dube

Monitoring Evaluation Accountability and Learning Coordinator, International Medical Corps 


\section{ABSTRACT}

Over the last decade Zimbabwe has made noteworthy progress in reducing both underweight and wasting in children under the age of five years, however one in four children in Zimbabwe is stunted. The rate in the decline of the number of children stunted still falls short of meeting the World Health Assembly target, and it goes without saying that effective, innovative community-based strategies are required by the government and development partners to accelerate the rate of stunting reduction. This paper presents experiences from using the Care Group approach for promoting improved maternal, infant and young child nutrition (MIYCN) and care based on lessons from the Amalima program, a seven-year United States Agency for International Development (USAID) Office of Food for Peace intervention. The Amalima program has been promoting Care Groups as a community and family centred approach to improve maternal and child nutrition in Zimbabwe. Care Groups are an innovative communitybased strategy that has been rolled out as part of the Amalima program activities in four food and nutrition insecure districts in Zimbabwe. The final programme evaluation suggested the program succeeded in increasing the exclusive breastfeeding rate and reducing levels of nutritional stunting among children under two years. In the present discussion paper, we present the key lessons learned and strategies we believe may have contributed to making Care Group implementation effective; we highlight the modifications that we made in Care Group implementation to ensure a context appropriate approach; and we discuss how Care Groups can be integrated into the Ministry of Health and Child Care structure. The critical factors for successful Care Group implementation have been grouped into five broad categories: conduct formative research; ensure context specific approaches \& adaptive management; leverage on social capital and cohesion; invest in human capital; prioritise quality assurance \& reviews.

\section{BACKGROUND}

Vast literature and evidence exist to highlight that adequate nutrition during the period from birth to two years of age is critical for a child's optimal growth, health and development and that childhood stunting is one of the most significant impediments to human development. The first 1000 days between conception and a child's second birthday has been identified as the most crucial window of opportunity for interventions to reduce stunting (Cusick \& Georgieff, 2019). Evidence suggests that stunting is largely irreversible after the first 1000 days, leading to an intergenerational cycle of poor growth and development, in which women who were stunted in childhood are at risk of remaining stunted as adults and tend to have stunted offspring (Georgiadis \& Penny, 2017). Stunting was endorsed as a key indicator for monitoring maternal, infant and young child nutrition by the World Health Organization (WHO, 2012; UNICEF, 2015).

\section{Stunting rates in Zimbabwe}

Whilst Zimbabwe has made commendable progress in reducing malnutrition in children under the age of five years, one in four children in Zimbabwe is stunted. (Food and Nutrition Council, 2018). The 2018 National Nutrition Survey indicates a national stunting prevalence rate of $26.2 \%$, which is considered high according to global standards (de Onis et al, 2019). The 
Government of Zimbabwe has aligned its commitment to the World Health Organization (WHO) target of a 40\% reduction in stunting by 2025. However, the latest projections anticipate that Zimbabwe will not meet it (WHO, 2019). Therefore, additional efforts are required by the government and development partners to accelerate the rate of stunting reduction.

The Zimbabwe National Nutrition Strategy promotes appropriate infant and young child feeding practices that contribute to improvements in early childhood nutrition, reduce undernutrition, and also contribute to improved health outcomes of children and mothers (Food and Nutrition Council, 2014). Whilst, several strides have been made to implement stunting reduction strategies including through multi-sectoral approaches which recognise nutrition specific and nutrition sensitive activities, much still needs to be done in reaching mothers and caregivers, and their families (influencers) with key messages for enhanced coverage, and strengthening the adoption of key behaviours to promote recommended infant and young child feeding practices (Ruel et al, 2013). In view of the fact that Zimbabwe will not meet the Sustainable Development Goal (SDG) 2 and the World Health Organization (WHO) target by 2025, impactful innovative, low cost community-based strategies are urgently needed to accelerate stunting reduction (ZNVR, 2017; WHO, 2019).

One of the important reasons for lack of progress has been the low population coverage of interventions. Perry et al (2015) state that expanding coverage of key interventions and achieving documented reductions in maternal, neonatal, and child mortality will require approaches that are low-cost, effective, and feasible at scale over the longer term. They recognise Care Groups as one example of the growing importance of participatory women's groups in improving maternal and child health and nutrition and share their experience with implementing Care Groups in Zimbabwe. This discussion paper presents some of our key lessons learned about Care Groups for improved maternal, infant and young child nutrition (MIYCN) from the Amalima program in rural Zimbabwe. We leverage on our experience of implementing Care Groups for 6 years on the USAID Food For Peace funded Amalima program which rolled out Care Groups across 4 food and nutrition insecure districts from 2014 to 2020. We describe the key ingredients for effective Care Group implementation, the modifications that we made in Care Group implementation, and how Care Groups can be integrated into the Ministry of Health and Child Care structure. Notably, the role of different stakeholders including governmental and non-governmental organisations, community-based organisations, and community leadership can be critical to supporting maternal and child health programs to address stunting. Evidence shows that Care Groups may provide a promising approach to significantly increase key child survival interventions and accelerate reductions in under five mortality rates (George et al, 2015). Companion articles looking at adolescent participation in Care Groups, and comparing diet quality amongst Care Group and non-Care Group participants are also being documented by the authors to contribute to the evidence on the effectiveness of the Care Group approach in Zimbabwe. 


\section{TACKLING THE STUNTING CHALLENGE IN ZIMBABWE: THE AMALIMA PROGRAM CARE GROUPS}

Care Groups are peer to peer support groups made up of pregnant or lactating women and caregivers of young children. The members are led by a trained facilitator, and meet on a regular basis. The objective of the group sessions is to impart knowledge, and skills and model practices for the adoption of key behaviours. The Care Group approach or model has the advantage of being able to reach a large number of caregivers using trained community health workers or volunteers. A study which evaluated the effectiveness of Care Groups concluded that Care Groups may provide a promising approach to significantly strengthen key child survival interventions and increase reductions in under 5 mortality rates (George et al, 2015). In Mozambique, a study demonstrated that using the Care Group model can reduce the level of global undernutrition in children at scale and at low cost and that the model shows sufficient promise to merit further rigorous testing and broader application (Davis et al, 2013). The economic benefits in terms of program cost and cost impact profile make the Care Group approach a promising strategy for improving child and maternal health in developing countries.

The Amalima program has been implementing Care Groups in Tsholotsho district in Matabeleland North Province and Gwanda, Bulilima and Mangwe districts in Matabeleland South Province. These four districts are in areas which are prone to low rainfall, drought and food insecurity. The goal of the Amalima program was to improve household food and nutrition security. The baseline study for the program was conducted in 2014 and the stunting rate was found to be at $31.7 \%$ and exclusive breastfeeding rate was at $44.9 \%$ (ICF, 2015).

In 2014, the USAID Food for Peace funded Amalima and ENSURE programs ${ }^{1}$ joined the growing list of non-governmental organizations implementing Care Groups world-wide. In Zimbabwe, this was the first time that Care Groups were implemented to scale to promote the adoption of recommended MIYCN practices at community level. A Care Group is a group of 10-15 volunteers, community-based health educators who regularly meet together with project staff for training and supervision. They are different from typical mother's groups in that each volunteer is responsible for regularly visiting 10-15 of her neighbours, sharing what she has learned and facilitating behaviour change at the household level. Care Groups create a multiplying effect to equitably reach every beneficiary household with interpersonal behaviour change communication (Food Security and Nutrition Network Social and Behavioral Change Task Force, 2014).

From program onset, the Care Group activities have been implemented collaboratively with the Ministry of Health and Child Care (MOHCC) structures. Firstly, at program onset the MOHCC was sensitised and engaged at National, Provincial and District level. Following this initial engagement, key MOHCC District level cadres ${ }^{2}$ were trained on the approach and engaged on how the Care Group activities could be best tailored for the context. Based on recommendations from discussions with MOHCC, it was proposed to implement the activities through utilisation of the Village Health Workers (VHW) ${ }^{3}$ as Care Group Volunteers. The

\footnotetext{
${ }^{1}$ The Amalima program is led by CNFA. Other partner organisations include International Medical Corps, The Manoff Group, Africare, Dabane Trust and ORAP.

${ }^{2}$ District Nutritionist, District Nursing Officer, and Community Nursing Sister

${ }^{3}$ Village Health Workers are community health volunteers under the Ministry of Health, who conduct community health activities with support from nurses from the local clinics/health centres
} 
MOHCC nurses from clinics/health centres received training on the Care Group approach and were engaged on how they could support the VHWs in rolling out the activities. The Care Groups create a multiplier effect ${ }^{4}$, reaching all households in a community at low cost (Perry et al 2015). On the Amalima program, over 2,000 Volunteers have been trained, with the same volunteers reaching over 100,000 households in rural areas using Care Groups to achieve the high coverage ${ }^{5}$ (IMPEL, 2020).

Nutrition social and behaviour change communication (SBCC) is a set of interventions that combines elements of interpersonal communication, social change and community mobilization activities, mass media, and advocacy to support individuals, families, communities, institutions, and countries to adopt and maintain high-impact nutrition-related practices. Effective nutrition SBCC seeks to increase the factors that encourage these behaviours while reducing the barriers to change. Amalima's Care Group Approach has been centred on nutritional social and behavioural change communication. Through a formative research, Amalima identified key MIYCN behaviours to be promoted for the program. Conducting formative research on key behaviours to be promoted was in line with what other researchers such as Perry et al. (2015) have suggested. According to USAID's Technical Guidance Brief for Effective At-Scale Nutrition Social and Behavior Change Communication, "virtually all the immediate and underlying causes of malnutrition are behavioural-influenced by the behaviours of individuals and their household members.”, (USAID, 2017). Table 1 shows some of the key behaviours identified through the formative research that are being promoted through the Care Groups.

Care Group trainings focused on promoting key behaviours to achieve optimal infant and young child feeding during the 1000-day window of opportunity period ${ }^{6}$. Figure 1

\section{Amalima Care Groups: a concise summary}

- The approach promotes optimal MIYCN and care practices in the first 1,000 days for prevention of chronic malnutrition

- A Care Group consists of up to 10 mothers or caregivers meeting once a month, and led by a trained Volunteer

- Amalima has 425 Care Group Volunteers (CGV), and 1,678 Lead Mothers (LM)

- Care Groups leverage on a multiplier effect to reach large numbers of mothers and caregivers with key messages each month

- CGVs and LMs use context and culturally appropriate materials in the local language - Amalima developed flipcharts and counselling cards informed by the UNICEF Infant and Young Child Feeding materials and Formative Research

- Mothers join Care Groups from pregnancy until the child is two years of age

- Meetings last up to two hours and are held in a central place that is easily accessible by foot for all.

- LMs share key messages through a participatory, interactive session with a behaviour-centred approach

- Session activities include community cooking demonstrations which promote dietary diversity utilising locally available foods

- LMs conduct a follow up home visit to the Care Group members to offer one on one tailored support, reach other family members with key messages and strengthen adoption of practices

- Some sessions are 'opened' up to men, mother in laws, grandmothers and other family members

- Ministry of Health Village Health Workers (VHW) were adopted as CGVs to avoid creating a parallel volunteer structure.

\footnotetext{
${ }^{4}$ Through the multiplier effect, 425 VHWs coached and mentored 1,678 Lead Mothers on key MIYCN messages. The trained Lead Mothers reached over 100,000 households in rural areas with key messages promoting adoption of recommended IYCF behaviors.

${ }^{5}$ Amalima program results for the period 2014 to 2020

${ }^{6}$ The 1000 days between a woman's pregnancy and her child's $2^{\text {nd }}$ birthday offer a unique window of opportunity to shape healthier and more prosperous futures. The right nutrition during this 1000-day window can have a profound impact on a child's ability to grow, learn, and rise out of poverty. It can also shape a society's long-term health, stability and prosperity.
} 
summarises the structure of the Amalima program Care Group model and illustrates its multiplier effect. Within the Amalima Care Group approach, Supervisors/ Nutrition Officers (NOs) who are paid program staff meet with, teach and support Promoters/Field Officers (FOs) who are also paid program staff, they in turn meet with, teach and support Care Group Volunteers (Ministry of Health Village Health Workers). Monthly, each Promoter is responsible for training and supportive supervision to a group of 5 to 12 Care Group Volunteers. The Care Group Volunteers (CGV) mentor and support Lead Mothers (LM), who are a lower-level cadre of community volunteers to work with groups of up to 10 mothers and caregivers (who are referred to as the Care Group).

Table 1: Examples of behaviours identified by formative research as needing improvement and being promoted through the Care Groups

\section{Infants 6-11 months}

- Mothers increase number of meals up to 3 times per day

- Mothers/caregivers give 1 nutritious snack per day from available food
- Mothers/caregivers feed animal source foods when available at least 2 times each week

- Mothers/caregivers enrich porridge with nuts, legumes and vegetables
Key Amalima AChievements

- Over 100,000 men and women have been reached through the Care Groups

- Exclusive Breastfeeding rate increased from 44.9 to $75.3 \%$

- Stunting reduced from 31.7 to $24.5 \%$ from 2014 to 2019; underweight reduced from 14.6 to $6.5 \%$ over the same period (IMPEL, 2020.)
The reduction in stunting levels from 31.7\% (baseline) to $24.5 \%$ (endline) is suggestive of the fact that mothers and caregivers were adopting the recommended infant and young child feeding (IYCF) practices promoted through the Care Groups. Based on the seven years of Care Group implementation, this discussion paper proposes factors for successful Care Group implementation.

\section{Suggested factors for successful Care Group implementation:}

\section{Formative Research}

- Conduct a Formative Research at inception as this is critical. The research helps the program identify the key behaviours to promote, and ensure that the recommended behaviours or practices are context and culturally appropriate. 


\section{Amalima Care Group model - district structure}

$1 \mathrm{NO}$

$$
\text { NO supervises }
$$

9 FO's

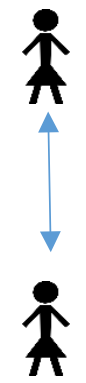

MOHCC

District

Nutritionist
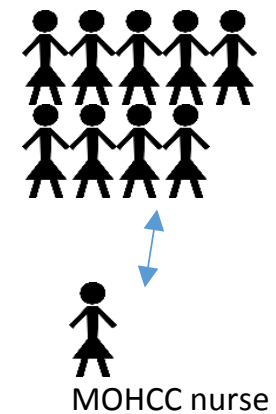

FO supervises

upto 12 CGVs

CGV supervises

upto $10 \mathrm{LMs}$

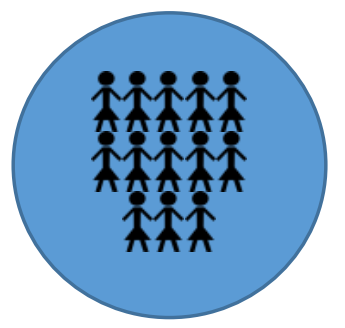

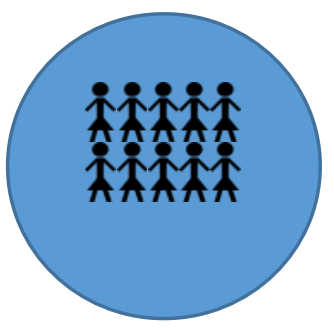

LM supports 10

CG members

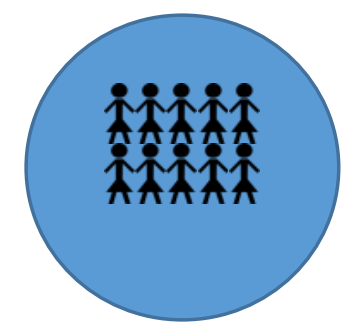

Figure 1: Amalima program Care Group model

NO- Nutrition Officer

FO- Field Officer

CGV- Care Group Volunteer

LM- Lead Mother

CG- Care Group

- As necessary, include other key topics as part of the curriculum to be covered during the Care Group sessions. The Amalima program mainstreamed key hygiene messages (5 critical times for handwashing, the faecal-oral route, and promotion of handwashing stations such as the 'tippy tap') as part of the curricular. Another message promoted the use of antiretroviral treatment for HIV-positive mothers and babies.

- The trials of improved practices (TIPS) methodology ${ }^{7}$ was utilized with groups of mothers from within the districts of operation.

\section{Context Specific Approaches \& Adaptive Management}

- The program had to look at limiting the workload for the volunteers to prevent volunteer fatigue. The program implementation areas are sparsely populated, and it was important to tailor the approach so as to reduce the burden of work on the CGVs and LMs. The program reduced the volunteer burden of work through the following ways:

- Care Group meetings take place once a month and not twice monthly, as is the case with other Care Group models.

- In the Amalima program, LM could only have up to 10 neighbour women per LM.

- Ensure that sessions do not take more than 2 hours at the most. On average, sessions should last 1 to 2 hours as neighbour women and their LM's have a lot of other chores that they do at home, and it is prudent to be mindful of this.

\footnotetext{
7 The TIPS methodology is a formative research technique which allows program planners to pretest the actual practices that a program will promote.
} 


\section{Be open to the views from the community}

- In a few wards in one of the project districts (Gwanda), the communities opted to have men taking up the role of 'Lead Mothers' as these communities were able to identify motivated men within their communities, who had a keen interest in infant and young child nutrition. These Lead Fathers were committed, and dedicated as they worked exceptionally hard surpassing expectations and adapting to their duties of conducting Care Group trainings and home visits.

- Invest in developing or adapting and modifying quality context appropriate materials in the local language. Visual teaching tools and participatory behaviour-change materials are essential. The Amalima program developed flipcharts and counselling cards in isiNdebele (the pre-dominant local language in the region). The materials were rigorously pre-tested to ensure acceptability and accuracy of terms used. This contributes hugely to the degree to which volunteers will 'own' the materials and use them.

- Focus on learning for improved programming (adaptive management) as you implement, as this will help you to make the necessary tweaks to ensure your approaches are context specific or appropriate. In addition, this will help address real gaps and challenges and improve program performance.

- It is important to mainstream gender sensitive approaches in the program. All Amalima program staff were trained on gender sensitive approaches, more importantly as the program was implemented in very patriarchal communities. In addition, CGVs and LM's were sensitised on key gender issues ${ }^{8}$ to strengthen gender sensitive programming.

- Analyse as you implement, and don't be afraid to be innovative. During the program design stage, there was no plan of developing a recipe book. However, during implementation it was recognized that mothers were shunning locally available foods, opting for more 'affluent' type of processed foods such as salty or sugary snacks which are unhealthy for young children. The team together with mothers, developed a recipe book that was to be used for the complementary feeding of children aged 6 to 23 months. Recipes created were enhanced (had their nutrient content modified) through the use of Nutri-Survey software. The selected recipes were translated into the local language of isiNdebele and compiled along with pictures (taken during the recipe creation) into a recipe book. The program distributed the recipe book to all Care Group members.

\footnotetext{
${ }^{8}$ As informed by the Amalima program's Gender Analysis
} 


\section{Social Capital and Cohesion}

- The Amalima Care Group approach recognises and epitomises the value of reaching the 'Sphere of influence around the mother', that is other family members (grandmothers, husbands, mother in laws etc) they are reached through a tailored Care Group session that is open for them to attend. They are also reached through the monthly home visit. This contributes hugely to ensuring an enabling environment around the mother - which may be an important precursor for adoption of promoted behaviours and practices. Evidence shows that people can change their behaviours to improve nutrition outcomes, especially when the environment in which they live and work supports those changes (Spring, 2017) - and this is a key strength of the Care Groups. Lead Mothers carry out monthly home visits to each Care Group member. Monthly contact between LM's and the mothers/caregivers is essential. This is promoted not only through the Care Group meetings, but also during the monthly home visit sessions which encourage strong interpersonal interface between the mother and the LM. The LM becomes an important part of the social support system for the neighbour women. In the Zimbabwean culture, it is not surprising to have family setups with a lot of 'other' family members living together as one household. These family members include grandmothers, grandfathers, aunts and uncles. Hence when these family members are present, they are invited to attend the home visit (Perry et al, 2015). As acknowledged by Aubel (2006), grandmothers are gatekeepers of indigenous knowledge and as such should be treated as the knowledge 'hard drives.' On the other hand, fathers/husbands are typically the household heads providing both financial and material resources for the up keep of their families. Against this background, and also the realization that when all family members are provided with the correct information by LMs, they (family members) can better support the mother during the period of infant and young child feeding. This is a critical way of enriching the mother's social capital and contributing to social cohesion at household and community level.

- The use of edutainment makes the Care Group sessions fun, interesting and engaging for the neighbouring women. Participatory learning with the use of role plays, songs and skits is important to convey key messages in a fun way that helps strengthen group cohesion. The Amalima program built in edutainment into the materials and made this an essential and mandatory component for Care Group session delivery. Peer to peer health and nutrition promotion (between neighbour women in the same Care Group) is key; through social cohesion mothers will naturally form relationships as they share experiences. The Care Group became a platform for mothers and caregivers to share their personal experiences, develop friendships, enrich their social capital through having a support network of other women to reach out to and give them a sense of empowerment. The sense of psychological safety is seen as central to the success of groups (Taylor and Mupeyiwa, 2019) including those promoting improved health and 
nutrition behavioural change. Psychological safety creates a context where individuals feel psychologically safe to engage, learn, and develop.

\section{Human Capital}

Resources are of paramount importance, to contribute to the success and quality of activities. A key resource for effective implementation of Care Groups is to ensure a project has adequate, trained human resources or staffing to ensure a robust structure is in place. The human resources include Supervisors, Promoters (the program used program staff ${ }^{9}$ as promoters), CGVs and LMs. In addition, resources for effective implementation of the Care Group program include financial resources to support quality program trainings.

- Recognise the volunteer is the backbone and is a key resource of the Care Group approach. The motivation of volunteers can make or break the success of the project. On the Amalima program, from program onset extrinsic motivation was critical to motivate the CGVs in carrying out the program activities. The program used job-aide related incentives ${ }^{10}$ to motivate the CGVs and LMs. Whilst extrinsic incentivisation has been critical in motivating the CGVs, intrinsic motivation has played a key role for sustained motivation of the volunteers. After the first year of program implementation, supervision and monitoring data showed that retaining LMs was considerably more difficult than retaining CGVs. This was to be expected given that the LMs were a new structure that was introduced by the program, and in some cases pre-conceived expectations for monetary incentives were not forthcoming. Amalima adjusted its approach for volunteer motivation by strengthening the intrinsic motivation of volunteers. For example, we worked to ensure that CGVs and LMs are recognised and appreciated by community leadership (traditional leaders include Councillors, Kraal Heads and Village Heads) and stakeholders. Amalima also placed a lot of value in sensitising community

\section{Integrating Care Groups into the MOHCC system}

From program onset, it was important to integrate Care Groups into the Ministry of Health system to help sustain the approach. The Amalima program did this in the following ways:

- Engaged MOHCC at all levels from project onset discussions were held with national level, Provincial level and District level MOHCC prior to implementation Shared the Formative Research findings with MOHCC, and involved $\mathrm{MOHCC}$ in developing and pre-testing Care Group materials

- MOHCC Village Health Workers were adopted as Care Group Volunteers as the project did not want to create a parallel volunteer structure

- MOHCC Nurses and Ward Nutrition Coordinators were engaged in a co-Promoter role as they worked alongside program Field Officers in training and supporting Care Group Volunteers in rolling out the Care Groups. Towards the last two years of the program, the Nurses and Ward Nutrition Coordinators took on a stronger/lead facilitation role whilst the Field Officers took on a reduced facilitation role.

Conducted supportive monitoring and supervision / mentorship visits together with key MOHCC cadres ${ }^{1}$. This enhanced MOHCC appreciation of the Care Group approach, recognising that strong linkages are a key factor for sustainability.

By working hand in glove with $\mathrm{MOHCC}$, the Amalima program Nutritionists received requests from MOHCC Provincial and District Nutritionists to scale up the activities to other nonprogram districts.

\footnotetext{
${ }^{9}$ Program Field Officers

${ }^{10}$ Bicycles to facilitate travel to support LMs, T-shirts with key messages, bags to carry training materials, hats, canvas shoes
} 
leadership on the value of the important work that CGVs and LMs were doing in their community such as promoting key health and nutrition practices for the prevention of malnutrition. This resulted in heightened community recognition of the volunteers. Interestingly, the CGVs and LMs place a lot of value in being recognised for the position that they hold, and being given a platform at community meetings to share key health and nutrition messages. Over time, the program implementers gradually reduced the extrinsic motivation by giving less and less of the job-aides whilst promoting increased intrinsic motivation of the volunteers (see Figure 2) as a way of building towards sustainability beyond the project life.

- Invest in capacity building on the key topics/messages to be delivered by the volunteers. The program trainings are carried out on a quarterly basis, where at the first instance project Nutrition Officers (Supervisors) train Field Officers (Promoters) on a set number of modules or sessions. The trained Field Officers then go on to train CGVs, who in turn train LMs. Once the LMs are trained, they deliver the training to mothers and caregivers (Neighbour women). The trainings have the advantage of reaching a large number of mothers and caregivers due to the cascade nature of the Care Group approach. Essentially, with the model, through one trained Field Officer the training can be cascaded to up to 1,000 mothers or caregivers on a monthly basis.

- Care Group lessons need to be delivered in a participatory, and not a didactic approach. This centres on ensuring that CGVs and LMs are trained on participatory, engaging adult training techniques. Amalima invested a lot of time in training the CGVs and LMs on adult learning techniques. The training cannot be a once-off training, it needs to be delivered on a pre-determined schedule (such as built into quarterly trainings for the CGVs and LMs) as part of their training on the key messages.

- Behaviour change is a process that takes time, and capacity building on counselling and negotiation skills cannot be overemphasised. The emphasis on behaviour change remains key throughout the Care Group training curricular, with use of behaviour change concepts informing Care Group session delivery.

- Strong collaboration with MOHCC from project onset, and throughout implementation was critical. This includes investing in capacity building for key MOHCC cadres. The Amalima program trained nurses and Ward Nutrition Coordinators on the key nutrition topics. The training was conducted in collaboration with MOHCC, and co-facilitated by the MOHCC District Nutritionists.

\section{Quality Assurance \& Reviews}

- $\quad$ Put in place an effective supportive monitoring system to ensure quality programming. The Amalima program conducted quarterly quality improvement verification checks to identify capacity gaps through monitoring of Care Group sessions using a modified quality improvement verification checklist (QIVC) ${ }^{11}$. One aspect of the Care Group model is the likely dilution of messaging from one volunteer level to another- as CGVs train/capacitate LMs and these in-turn capacitate neighbouring women. Having the ${ }^{11}$ The Amalima program developed a QIVC checklist in the local language, isiNdebele adapted from the QIVC in the Technical and
Operational Performance Support (TOPS) Program Care Group toolkit 
QIVC in place is one of the essential criteria in applying the Care Group approach to ensure regular supervision of volunteers. Supportive supervision has also been identified by Hill et al (2014) as being key in the implementation of community health worker programs. For the Amalima programme, CGVs and LMs through supportive supervision have reported feeling supported and this in-turn has contributed to their intrinsic motivation to some extent.

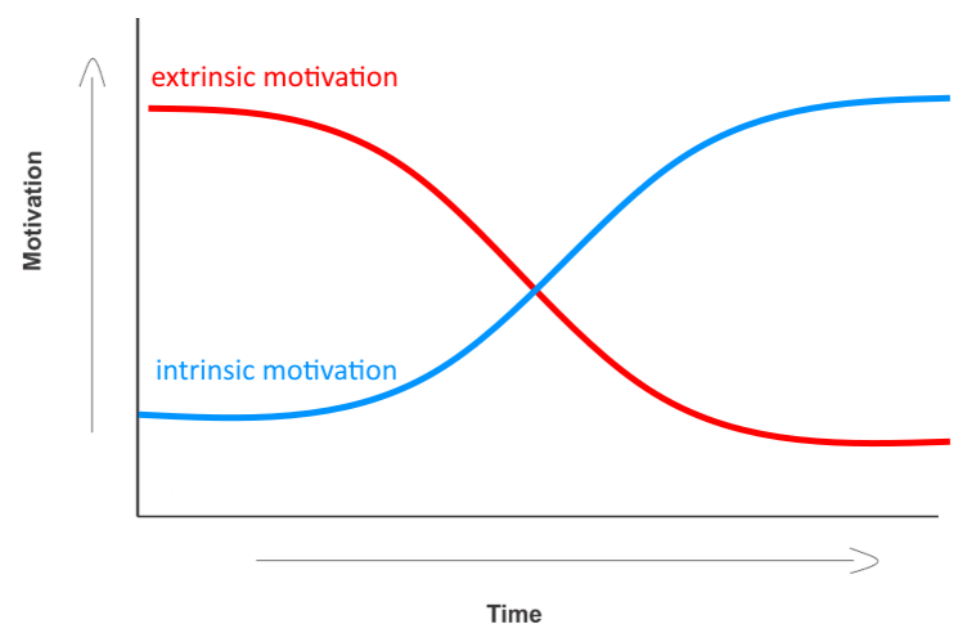

Figure 2: Volunteer motivation - extrinsic versus intrinsic motivation

- Provide platforms for learning, and sharing. Quarterly review meetings which bring the volunteers together for capacity building on gaps identified through the QIVC. The Quarterly review meetings are facilitated by MOHCC nurses and Ward Nutrition Coordinators in collaboration with Promoters (Field Officers). This is in line with Amalima's sustainability strategy which sees MOHCC cadres supporting the trained volunteers after the project ends. The quarterly review meetings give the volunteers a platform to learn from each other, and share experiences. In addition, it gives them an enhanced understanding of the vastness of the stunting problem, giving them heightened appreciation for the program objective to reduce malnutrition in their communities. Overall, the platform presented an opportunity for both groups of volunteers to receive the same type of training and messages, solve problems and plan for routine activities (Vernon et al, 1994).

\section{CONCLUSIONS}

There is a substantial body of literature documenting experiences on implementing Care Groups; this discussion article adds to experiences on operationalising the approach in a rural setting in Zimbabwe. The Amalima Care Group interventions contributed to a reduction in stunting by 7 percentage points in rural Zimbabwe. Based on the Amalima program experience, the Care Group approach presents a promising alternative to current strategies for delivering key nutrition messages geared for behavioural change in rural Zimbabwean communities. The programme identified and leveraged on five success factors (Figure 3) in the implementation of the Care Group model in Zimbabwe. It is also encouraging that Care Groups in Zimbabwe were endorsed by the MOHCC 


\section{Amalima Care Group: Factors for successful Care Group implementation}

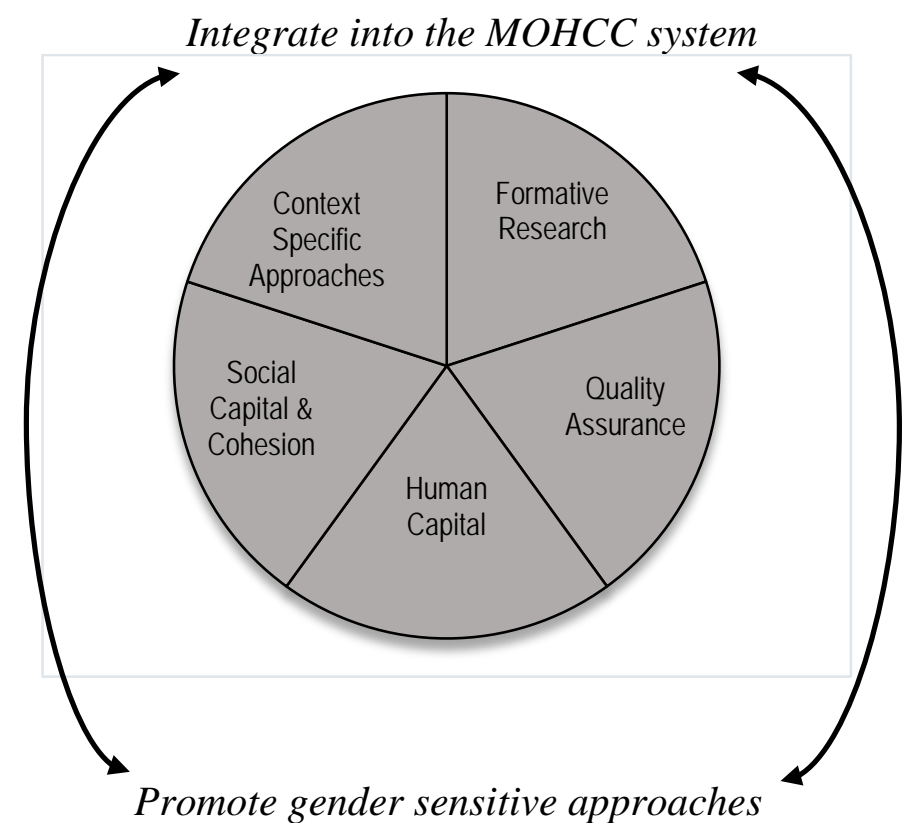

Figure 3: Factors for successful Care Group implementation - this presents a summary of the 'ingredients' for successful care group implementation as informed by the Amalima program experiences.

as the vehicle of choice for promoting appropriate infant and young child feeding and care practices that contribute to improvements in early childhood nutrition, reduce under-nutrition, and also contribute to improved health outcomes for children and mothers. At a National Care Group Model Seminar held in the country, there was consensus to enhance the adoption of the Care Group Model in Food and Nutrition Security Programming ${ }^{12}$. This discussion paper contributes to the best practices in promoting Care Groups in Zimbabwe given the urgency for promising strategies that reach large populations in an effort to accelerate stunting reduction and to address the slow progress being made against the WHO targets. Even though the costs of implementing the Care Group model may seem large in the beginning of implementation, its very success when effectively implemented lies in its multiplier effect. Implementers need to understand the communities and thus adapt the approach as necessary and ensure context appropriateness, capacity building of volunteers and motivation (intrinsic and extrinsic), strengthen Care Group cohesion, promote good quality assurance systems and incorporate Care Groups into the MOHCC system to help make them sustainable.

\footnotetext{
${ }^{12}$ Communique on Care Group Model for delivery of Community Nutrition Interventions, Declarations on the National Nutrition ENSURE Care Group Model Seminar held at Cresta Lodge, Harare, Zimbabwe from 27 - 28 August 2018
} 
Disclaimer: The views and opinions of the authors expressed herein do not necessarily state or reflect those of USAID or the United States Government.

\section{Acknowledgements:}

This discussion paper was facilitated through the USAID Food For Peace funded Amalima Development Food Security Activity in Zimbabwe. The authors would like to recognize the invaluable input from the following reviewers: David Brigham, Amalima Chief of Party (CNFA), Dr Nqobile Ncube, Amalima Deputy Chief of Party (ORAP), Dr Themba Nduna, Nutrition Adviser (USAID, Zimbabwe).

The Ministry of Health and Child Care (MoHCC), Zimbabwe for their support in prioritising improved maternal, infant and young child nutrition in Zimbabwe.

The Amalima DFSA team for all their hard work and the programme volunteers (Care Group Volunteers and Lead Mothers) whose dedication and diligence continually contributes to improved nutrition and health in Zimbabwe. 


\section{REFERENCES}

Aubel J. (2006). Grandmothers Promote Maternal and Child Health: The Role of Indigenous Knowledge Systems' Managers. Indigenous Knowledge (IK) Notes; No. 89. World Bank, Washington, DC. (C) World Bank. https://openknowledge.worldbank.org/handle/10986/10745 License: CC BY 3.0 IGO.

Cussick S and Georgeiff MK. (2019). The first 1,000 days of life: The brain's window of opportunity.

https://www.unicef-irc.org/article/958-the-first-1000-days-of-life-the-brains-window-ofopportunity.html

Davis TP, Wetzel C, Avilan EH, de Mendoza Lopes C, Chase RP, Winch PJ, Perry HB. (2013). Reducing child global undernutrition at scale in Sofala Province, Mozambique, using Care Group Volunteers to communicate health messages to mothers. Global Health: Science and Practice 1(1):35-51; https://doi.org/10.9745/GHSP-D-12-00045

de Onis M, Borghi E, Arimond M, Webb P, Croft T, Saha K. et al. (2019). Prevalence thresholds for wasting, overweight and stunting in children under 5 years. Public Health Nutrition 22(1):175-179. doi: 10.1017/S1368980018002434

Food and Nutrition Council. (2014). The Zimbabwe Nutrition Strategy.

https://extranet.who.int/nutrition/gina/sites/default/files/ZWE\%202014\%20National\%20Nutri tion\%20Strategy.pdf

Food and Nutrition Council of Zimbabwe. (2018). Zimbabwe National Nutrition Survey 2018. http://fnc.org.zw/wp-content/uploads/2019/01/Zimbabwe-2018-National-Nutrition-Survey$\underline{\text { Report.pdf }}$

Food Security and Nutrition Network Social and Behavioral Change Task Force. (2014). Care Groups: A Training Manual for Program Design and Implementation. Washington, DC: Technical and Operational Performance Support Program. 
George CM, Vignola E, Ricca J, Davis T, Perin J, Tam, Y and Perry, H. (2015). Evaluation of the effectiveness of Care Groups in expanding population coverage of Key child survival interventions and reducing under-5 mortality: A comparative analysis using the lives saved tool (LiST). BMC Public Health 15: 835 https://dx.doi.org/10.1186\%2Fs12889-015-2187-2

Georgiadis, A and Penny, ME. (2017). Child undernutrition: opportunities beyond the first 1000 days. The Lancet 2: e399

Hill Z, Dumbaugh M, Benton L, Källander K, Strachan D, Ten Asbroek A, Tibenderana J, Kirkwood B, Meek S. (2014). Supervising community health workers in low-income countries-a review of impact and implementation issues. Global health action 7(1):24085. DOI: 10.3402/gha.v7.24085

ICF. (2015). Baseline Study of the Title II Development Food Assistance Programs in Zimbabwe, June 3, 2015.

https://www.usaid.gov/sites/default/files/documents/1866/Zimbabwe\%20Baseline\%20Study \%20Report\%2C\%20June\%202015.pdf

IMPEL. (2020). Final Performance Evaluation of the Amalima Development Food Assistance Project in Zimbabwe (Vol. 1). Washington, DC: The Implementer-Led Evaluation \& Learning Associate Award.

Perry H, Morrow M, Borger S, Weiss J, Decoster M, Davis T, Ernst P. (2015a). Care Groups 1: An Innovative Community-Based Strategy for Improving Maternal, Neonatal and Child Health in Resource-Constrained Settings, Global Health: Science and Practice. 3(3): 358-369. https://dx.doi.org/10.9745\%2FGHSP-D-15-00051

Perry H, Morrow M, Davis T, Borger S, Weiss J, DeCoster M, et al. (2015b). Care Groups II: A summary of the child survival outcomes achieved in high-mortality, resource-constrained settings using volunteer health workers. Glob Health Sci Pract 3(3):370-381.

Ruel MT, Alderman H, The Maternal and Child Study Group. (2013). Nutrition-Sensitive interventions and programmes: how can they help to accelerate progress in improving maternal and child nutrition? The Lancet. 382(9891): 536-551 
https://doi.org/10.1016/S0140-6736(13)60843-0

SPRING. 2017. Moving Nutrition Social and Behavior Change Forward: Lessons from the SPRING Project.

https://www.springnutrition.org/sites/default/files/publications/briefs/spring_sbc_learning_brief.pdf

Taylor J and Mupeyiwa J. (2019). Prioritizing Psychological Safety as a Driver of Food Security and Resilience. https://www.agrilinks.org/post/prioritizing-psychological-safetydriver-food-security-and-resilience

UNICEF. (2012). Infant and Young Child Feeding: Counselling Cards for Community Workers.

https://www.unicef.org/nutrition/files/counseling_cards_Oct._2012small.pdf

United Nations Children's Fund. (2015). UNICEF's approach to scaling up nutrition for mothers and their children. Discussion paper. Programme Division, UNICEF, New York, June 2015.

https://www.unicef.org/nutrition/files/Unicef_Nutrition_Strategy.pdf

USAID. (2017). USAID’s Technical Guidance Brief for Effective At-Scale Nutrition Social and Behavior Change Communication.

https://www.usaid.gov/sites/default/files/documents/1864/at-scale-nutritionSBCC-technicalguidance-brief-edit-508.pdf

Vernon R, Staunton A, Garcia M, Arroyo JJ, Rosenberg R. (1994). A test of alternative supervision strategies for family planning services in Guatemala. Stud Fam plan. 25: 232-8

WHO. (2019). WHA Global Nutrition Targets 2025: Stunting Policy Brief. https://www.who.int/nutrition/topics/globaltargets_stunting_policybrief.pdf

Zimbabwe Voluntary National Review. (2017). Voluntary National Review of SDGs for the High Level Political Forum.

https://sustainabledevelopment.un.org/content/documents/15866Zimbabwe.pdf 
World Nutrition 2020;11(2):90-107 